\title{
Microsurgical Decompression for Peroneal Nerve Entrapment Neuropathy
}

\author{
Daijiro Morimoto, ${ }^{1,2}$ Toyohiko IsU, ${ }^{2}$ Kyongsong KIM, ${ }^{3}$ Atsushi SugawArA, ${ }^{4}$ \\ Kazuyoshi YAMAZAKI, ${ }^{2}$ Yasuhiro CHIBA, ${ }^{2}$ Naotaka IWAMOTO, ${ }^{2}$ Masanori IsOBE, ${ }^{2}$ \\ and Akio MORITA ${ }^{1}$ \\ ${ }^{1}$ Department of Neurosurgery, Nippon Medical School, Tokyo; \\ ${ }^{2}$ Department of Neurosurgery, Kushiro Rosai Hospital, Kushiro, Hokkaido; \\ ${ }^{3}$ Department of Neurosurgery, Nippon Medical School Chiba Hokusou Hospital, Inzai, Chiba; \\ ${ }^{4}$ Department of Neurosurgery, Iwate Medical University, Morioka, Iwate
}

\begin{abstract}
Peroneal nerve entrapment neuropathy (PNEN) is one cause of numbness and pain in the lateral lower thigh and instep, and of motor weakness of the extensors of the toes and ankle. We report a less invasive surgical procedure performed under local anesthesia to treat PNEN and our preliminary outcomes. We treated 22 patients (33 legs), 7 men and 15 women, whose average age was 66 years. The mean postoperative follow-up period was $\mathbf{4 0}$ months. All patients complained of pain or paresthesia of the lateral aspect of affected lower thigh and instep; all manifested a Tinel-like sign at the entrapment point. As all had undergone unsuccessful conservative treatment, we performed microsurgical decompression under local anesthesia. Of 19 patients who had undergone lumbar spinal surgery (LSS), 9 suffered residual symptoms attributable to PNEN. While complete symptom abatement was obtained in the other 10 they later developed PNEN-induced new symptoms. Motor weakness of the extensors of the toes and ankle [manual muscle testing (MMT) 4/5] was observed preoperatively in 8 patients; it was relieved by microsurgical decompression. Based on self-assessments, all 22 patients were satisfied with the results of surgery. PNEN should be considered as a possible differential diagnosis in patients with L5 neuropathy due to lumbar degenerative disease, and as a causative factor of residual symptoms after LSS. PNEN can be successfully addressed by less-invasive surgery performed under local anesthesia.
\end{abstract}

Key words: peroneal nerve, entrapment neuropathy, microsurgical decompression, clinical outcome

\section{Introduction}

Peroneal nerve entrapment neuropathy (PNEN) is one cause of numbness and pain in the lateral lower thigh and instep, and of motor weakness of the extensors of the toes and ankle. Entrapment around the fibular head is the most common cause of PNEN. In earlier reports, some entrapment sites and good postoperative treatment outcomes were reported. ${ }^{1-8)}$ However, as these operations were performed under general anesthesia, the risk for excessive procedures to address the possible entrapment sites cannot be ruled out. We report a less invasive surgical procedure performed under local anesthesia and our preliminary outcomes. We document our intraoperative findings, clinical results, and the clinical features of PNEN in our series.

Received January 6, 2015; Accepted May 22, 2015

\section{Materials and Methods}

We treated 22 patients with PNEN surgically; one leg was affected in 11- and both legs in the other 11 patients (total 33 legs). They were 7 men and 15 women ranging in age from 47 to 89 years (average 66 years). The symptom duration from onset to treatment averaged 13.2 months (range 2-84 months), the mean postoperative follow-up period was 40 months (range 34-46 months). In some patients, the pretreatment observation period was short and our decision to intervene surgically was based on their pain intolerance and/or their demand for treatment. Our diagnosis was primarily based on clinical symptoms and nerve conduction studies (NCSs). In NCS, the peroneal nerve (PN) was stimulated on the popliteal fossa, under the fibular head and on the ankle joint, and the nerve conduction velocity (NCV) and the compound muscle action potential 
(CMAP) amplitude of the extensor hallucis longus were evaluated. All patients complained of pain or paresthesia of the lateral aspect of the affected lower thigh and instep; all presented with a positive Tinel-like sign at the entrapment point.

Based on radiological studies and clinical symptoms such as no symptoms at the thigh and no positive sign during the straight leg-raising- and/or Kemp test, we ruled out coexisting lumbar spine disease. All patients had undergone unsuccessful conservative treatment. We excluded patients who obtained pain relief by conservative treatment, refused surgical treatment, were ineligible for surgery due to their general condition, and patients with dementia. By self-assessment they reported postoperative changes in their symptoms and their degree of satisfaction (satisfied, acceptable, dissatisfied).

\section{Surgical Method}

With the patients in the lateral position and the side of the lesion uppermost, we performed microsurgical decompression of the affected common peroneal nerve (CPN) under local anesthesia without a proximal tourniquet. To confirm symptom improvement intraoperatively we delivered no sedatives. A longitudinal skin incision measuring approximately $3 \mathrm{~cm}$ was made along the CPN around the fibular neck and continued down through the subcutaneous tissues to expose the superficial fascia between the superficial head of the peroneus longus and the gastrocnemius. The superficial fascia was carefully opened to expose the CPN which is located under the fibrous band between the superficial head of the peroneus longus and the soleus. This fibrous band was dissected distally to the CPN (approximately $2 \mathrm{~cm}$ ) without transection of the superficial head of the peroneus longus. Upon release the patients usually reported symptom relief; the Tinel-like sign disappeared. The superficial head of the peroneus longus muscle was retracted medially to inspect the CPN running to the peroneal tunnel and to rule out entrapment in the tunnel. During neurolysis, as little as possible of the perineural fat tissue was removed to prevent postoperative adhesion. Skin closure was without fascia closure. Just after surgery the patients were allowed to walk without cast immobilization; they were discharged the next day.

\section{Results}

Of the 22 patients, 19 (86.4\%) had previously undergone lumbar spinal surgery (LSS) and 5 $(22.7 \%)$ had medically-treated diabetes mellitus. Postoperative residual symptoms due to PNEN were reported by 9 patients $(47.4 \%)$. While the other 10 experienced complete postoperative symptom improvement, they subsequently developed PNENinduced new symptoms. These gradually progressed without inducible factors such as the previous orthopedic surgery, trauma to the ipsilateral leg, or external sources such as a leg cast with the knee in hyperextension, prolonged knee bracing, habitual leg crossing, or their sleeping position. In 14 of the 22 patients $(63.6 \%)$, the symptoms were exacerbated by walking and similar to the intermittent claudication seen in patients with lumbar degenerative disease (LDD). Preoperative NCS had shown reduced CMAP amplitudes and NCVs in $9(40.9 \%)$ of 22 patients without conduction block; in 13 (59.1\%) these findings were normal. Motor weakness of the extensors of the toes and ankle (MMT 4/5) was observed in 8 (36.4\%) patients.

Decompression with dissection of the fibrous band between the superficial head of the peroneus longus and the soleus, considered the primary site of CPN compression, produced symptom relief in all 22 patients. In this series there were no mass lesions around the CPN such as neurinoma, ganglion, and tumor. There were no surgery-related complications and no patients reported postoperative symptom recurrence during the follow-up period. Based on self-assessments, all 22 patients were satisfied with the results of surgery.

\section{Illustrative Case}

A 60-year-old woman with a 5-year history of numbness of the lateral aspect of the left leg and the dorsal foot and slight motor weakness of ankle dorsoflexion (MMT 4/5) had been conservatively treated with medications. However, her symptoms gradually worsened and were exacerbated by walking $(100 \mathrm{~m})$ and standing (10 min). Lumbar radiological studies revealed no causative abnormalities; electrophysiological examination disclosed no conduction block on the $\mathrm{PN}$. The diagnosis of PNEN at the peroneus tunnel was made based on her clinical symptoms and the detection of a positive Tinel-like sign at the peroneus tunnel. As conservative therapies had failed and her activities of daily living were affected we performed microsurgical decompression for left PNEN under local anesthesia.

The CPN was compressed by the fibrous band between the superficial head of the peroneus longus and the soleus (Figs. 1, 2). After its dissection we observed bulging of the CPN (Fig. 3). Upon manual direct compression of the CPN she reported symptom relief and disappearance of the radiating pain. Her postoperative course was uneventful and 


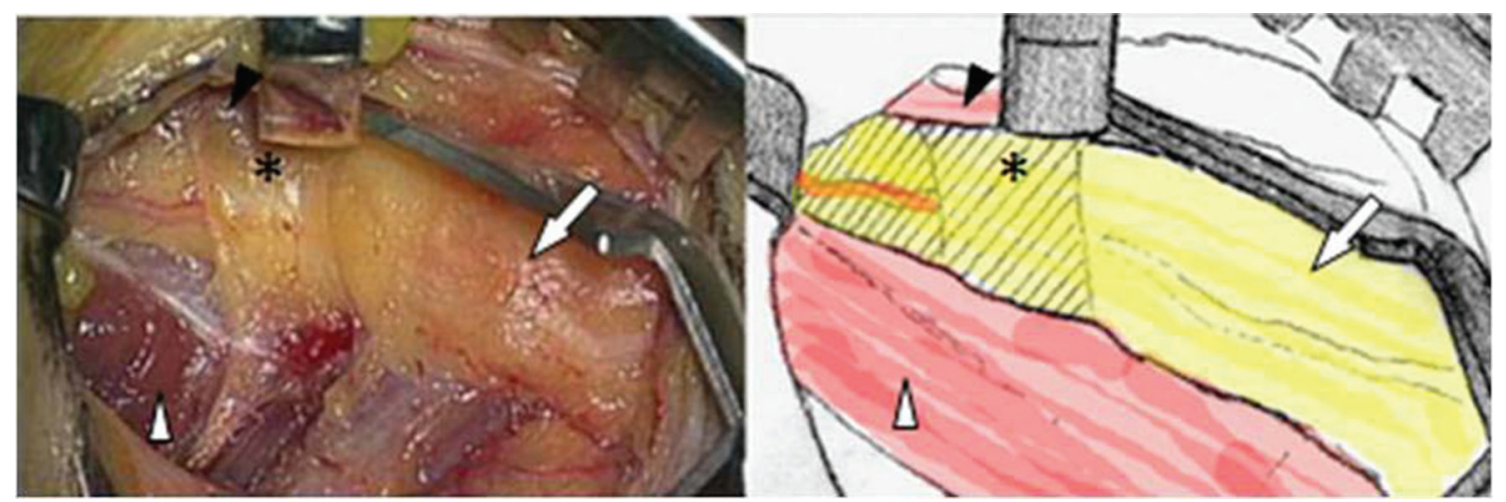

Fig. 1 Photograph and schema demonstrating compression of the peroneal nerve (arrow) by the fibrous band (star) between the peroneus longus (black arrowhead) and the soleus (white arrowhead). The peroneus longus was retracted medially.

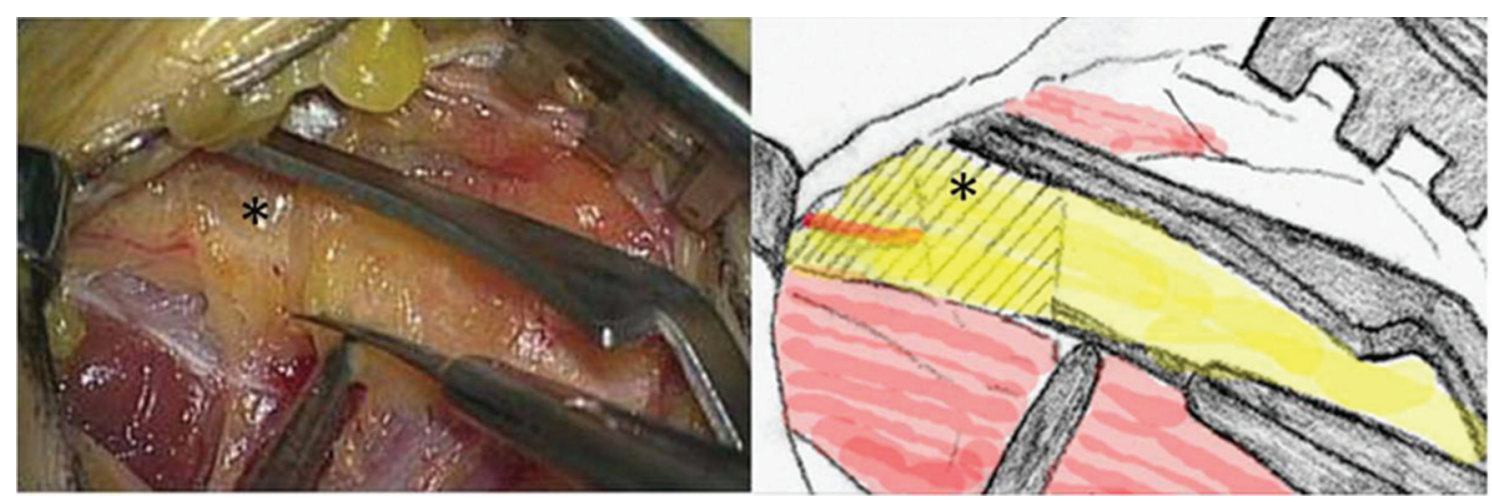

Fig. 2 The fibrous band (star) is sharply dissected with microscissors.

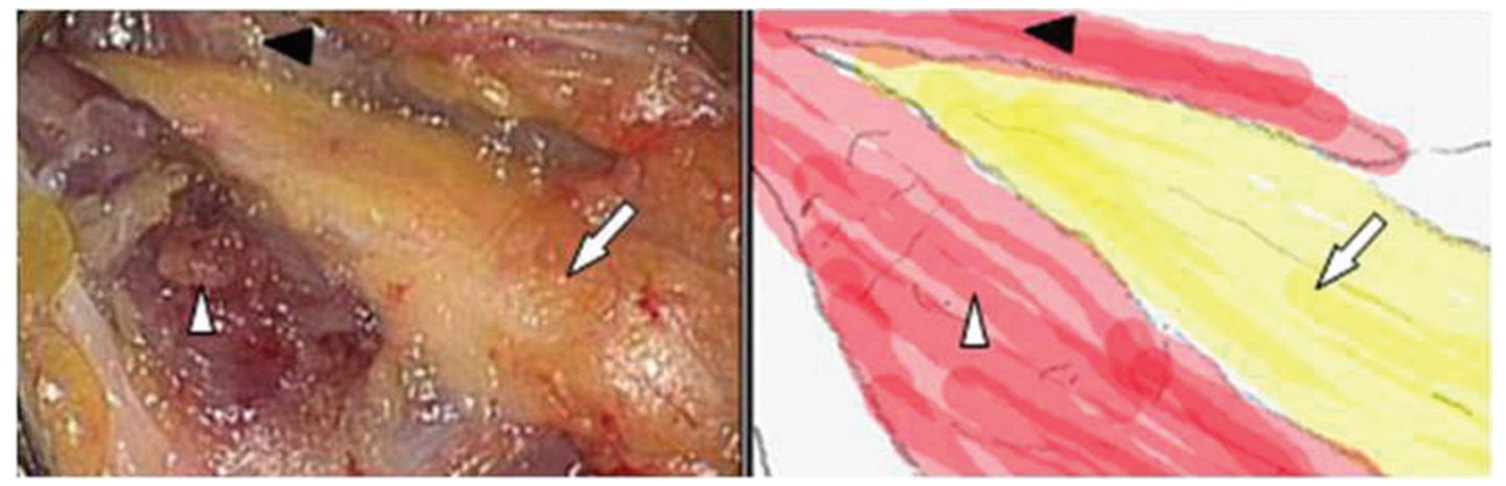

Fig. 3 After 2-cm dissection of the fibrous band, the bulging peroneal nerve (arrow) is decompressed. Black and white arrowheads indicate the peroneus longus and soleus, respectively.

her symptoms disappeared and had not recurred 12 months later.

\section{Discussion}

In patients with PNEN, the common, superficial, and deep PNs are compressed around the fibular head and good postoperative treatment outcomes have been reported. ${ }^{1-8)}$ The intermuscular septum, ${ }^{8)}$ peroneal muscle fascia, ${ }^{3,8)}$ confluence of the origin of the soleus and peroneus, ${ }^{3,7)}$ entrance of the fibular tunnel, ${ }^{6)}$ fibrous band surface of the deep head of the peroneus longus, ${ }^{7)}$ and the fibrous band deep to the peroneus longus ${ }^{3,7)}$ are the previously-reported entrapment sites. These single or multiple entrapment points were identified based on intraoperative or 
cadaveric findings and surgery was performed under general anesthesia. As the correlation between some of the proposed entrapment points and the clinical symptoms is unreliable, excessive procedures may be used during CPN decompression. As we performed surgery under local anesthesia and without a proximal tourniquet, we could confirm symptom improvement and disappearance of the Tinel-like sign intraoperatively. This helps to avoid excessive dissections and procedures, reduces the incidence of surgical complications, and does not require local immobilization of the knee. The major element of CPN compression is the fibrous band between the superior head of the peroneus longus and the soleus. Our surgical procedure helps to identify one of the etiologies of PNEN.

The dynamic factors of PNEN have been documented by Fabre et al. ${ }^{3)}$ The causative factor in their two patients was dynamic; one patient was a triathlete and the other a soccer player; their bilateral PN was pushed against the fibrous arch by their welldeveloped leg muscles. The authors speculated that repeat flexion and extension of the knee during sports activities resulted in irritation of the nerve as it moved within the tight arch. In 14 (63.6\%) of our patients the symptoms were exacerbated by walking and mimicked the intermittent claudication seen in patients with LDD. As none of our patients were athletes or had the well-developed leg muscles of athletes, we considered PNEN due to dynamic entrapment unlikely.

The superficial and deep PNs arise from the CPN at the peroneal tunnel. The superficial PN innervates the peroneus longus and the peroneus brevis, and deep PNs innervate the tibialis anterior, the extensor hallucis longus, and the extensor digitorum longus. Therefore, motor weakness of these muscles can be induced by PNEN. In eight of our patients (36.4\%) their preoperative motor weakness of ankle dorsoflexion disappeared postoperatively (from MMT 4/5 to MMT $5 / 5$ ). PNEN can induce not only drop foot and severe motor weakness but also mild motor weakness. ${ }^{3)}$ As the sensory disturbance and motor deficit are similar to L5 neuropathy, PNEN should be considered as a differential diagnosis in patients with L5 neuropathy attributable to LDD. Of our 22 patients, 19 (86.4\%) had previously undergone LSS; PNEN was the cause of their residual or relapsing symptoms. Although the incidence of PNEN after LSS is unclear in our series because we did not separately evaluate the patients who had undergone LSS, we suggest that PNEN be considered as a causative factor of these symptoms after LSS, and as a treatable condition.

Our diagnosis of PNEN was primarily based on clinical symptoms and the positive Tinel-like sign in the area of the peroneus tunnel. Because our patients' symptoms were typical for PNEN we did not induce PN block. Although this method is useful for the detection of the referred nerve, it may not help to differentiate between the L5 and the PN because the latter is innervated by the L5 nerve. According to Fabre et al.,3) a positive Tinellike sign was elicited in 58 of their 60 patients (96.7\%) with PN entrapment. Electrophysiological examinations, necessary for the diagnosis of entrapment neuropathies, have been suggested to be useful to diagnose PNEN. ${ }^{9,10)}$ We found that the sensitivity of preoperative NCS was not high enough to identify the site of CPN entrapment at the peroneus tunnel; these studies yielded negative findings in 13 (59.1\%) of our patients. Rather, the presence of characteristic symptoms and a positive Tinel-like sign at the peroneus tunnel are useful, simple, and convenient for distinguishing between L5 neuropathy and PNEN induced by CPN entrapment.

All the 22 patients obtained symptom relief after PNEN surgery and none suffered postoperative symptom recurrence. We think that our technique for diagnosing PNEN and our less invasive surgical procedure contributed to our satisfactory treatment outcomes. In earlier series, $50-87 \%$ of operated patients reported significant improvement in motor functions. ${ }^{3-6,11)}$ Ramanan et al. ${ }^{5)}$ suggested that good results can be obtained if surgery is performed within 12 months of the onset of motor dysfunction. All eight of our patients with motor dysfunction reported postoperative improvement; preoperatively, none manifested severe motor paresis and their motor weakness was low-grade (MMT 4/5). Consequently, they were not cognizant of their motor weakness until the diagnosis of PNEN. Patients with low-grade motor weakness may experience improved motor function postoperatively, even when their symptoms were present longer than 12 months before the diagnosis of PNEN.

Our study has some limitations. The number of patients was small and the postoperative follow-up period was relatively short (mean 40 months). Nonetheless, none of our patients suffered postoperative pain recurrence. To assess the eventual rate of recurrence resulting from scar formation or adhesions, long-term follow-up studies are needed.

\section{Conclusion}

PNEN should be considered as a differential diagnosis of L5 neuropathy attributable to LDD, and as a causative factor of residual symptoms after LSS. PNEN can elicit intermittent claudication as is seen in patients with LDD. PNEN patients who do not 
respond to conservative therapy can be successfully treated by less-invasive surgery performed under local anesthesia.

\section{Conflicts of Interest Disclosure}

None.

\section{References}

1) Kopell HP, Thompson WAL: Peroneal Entrapment Neuropathies. Baltimore, Williams and Wilkins, 1963, pp 34-47

2) Maudsley RH: Fibular tunnel syndrome. J Bone Joint Surg Br 49: 384, 1967

3) Fabre T, Piton C, Andre D, Lasseur E, Durandeau A: Peroneal nerve entrapment. J Bone Joint Surg Am 80: 47-53, 1998

4) Vastamäki M: Decompression for peroneal nerve entrapment. Acta Orthop Scand 57: 551-554, 1986

5) Ramanan M, Chandran KN: Common peroneal nerve decompression. ANZ J Surg 81: 707-712, 2011

6) Humphreys DB, Novak CB, Mackinnon SE: Patient outcome after common peroneal nerve decompression. J Neurosurg 107: 314-318, 2007
7) Dellon AL, Ebmer J, Swier P: Anatomic variations related to decompression of the common peroneal nerve at the fibular head. Ann Plast Surg 48: 30-34, 2002

8) Nogueira MP, Paley D: Prophylactic and therapeutic peroneal nerve decompression for deformity correction and lengthening. Oper Tech Orthop 21: 180-183, 2011

9) Berry H, Richardson PM: Common peroneal nerve palsy: a clinical and electrophysiological review. J Neurol Neurosurg Psychiatr 39: 1162-1171, 1976

10) Mitra A, Stern JD, Perrotta VJ, Moyer RA: Peroneal nerve entrapment in athletes. Ann Plast Surg 35: 366-368, 1995

11) Thoma A, Fawcett S, Ginty M, Veltri K: Decompression of the common peroneal nerve: experience with 20 consecutive cases. Plast Reconstr Surg 15: 1183-1189, 2001

Address reprint requests to: Daijiro Morimoto, MD, $\mathrm{PhD}$, Department of Neurosurgery, Nippon Medical School, 1-1-5 Sendagi, Bunkyo-ku, Tokyo 113-8603, Japan.

e-mail: dai_sampo@yahoo.co.jp 